\title{
Right ventricular needle embolus in an injecting drug user: the need for early removal
}

\author{
D L Ngaage, M E Cowen
}

\begin{abstract}
This case report describes an unusual cardiac complication in a 22 year old, female injecting drug user. The retention of two fractured injection needles at the site of intravenous injection in the groin, and the subsequent embolisation of one to the right ventricle, predisposed to recurrent local and systemic infections, and endocarditis. Two years later, the needle was completely embedded in the wall of the right ventricle and not suitable for transvenous removal. Removal of the retained and/or embolised needle at an earlier stage would have precluded these complications.

(Emerg Med f 2001;18:500-501)
\end{abstract}

Keywords: right ventricle; needle embolus; recurrent endocarditis

The communal and repeated use of injection needles predisposes injecting drug users to several complications. The commonly reported cardiovascular complication in this group of patients is infective endocarditis. ${ }^{12}$ A right ventricular needle embolus has not yet been reported in injecting drug users.

We report and discuss the treatment of this unusual cardiovascular complication in an injecting drug user.

\section{Case report}

A 22 year old female injecting drug user was admitted for bilateral VATS decortication. She had staphylococcal pneumonia three months before admission and subsequently, developed bilateral empyema. Two years previously, she fractured two injection needles in her left groin and concealed it. This was complicated by recurrent left groin infections and a deep vein thrombosis (DVT) in the left leg. Six months afterwards, she developed a blood culture positive staphyloccocal endocarditis. Her chest radiograph (fig 1) and transthoracic echocardiography demonstrated a needle lying free in the right ventricle (RV) seeded with vegetations. She refused transvenous removal of the needle embolus, and in the next 18 months she had three episodes of bacterial endocarditis.

She is hepatitis C positive, a heavy user of heroine and cocaine, and an ex-convict.

She has been admitted and treated numerous times in the accident and emergency department for illicit drug overdose, and on two occasions for organic depression and attempted suicide with paracetamol overdose.

On admission, she was dyspneic on minimal exertion with an oxygen saturation of $87 \%, \mathrm{PO}_{2}$

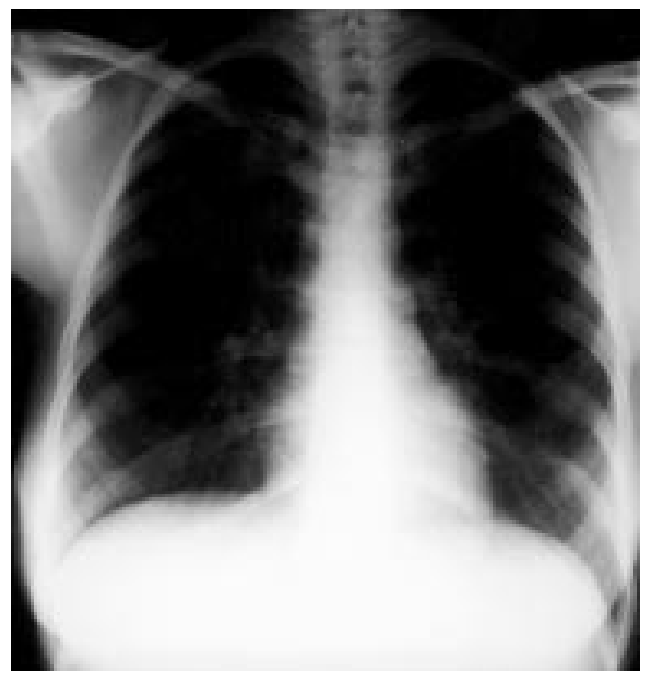

Figure 1 A plain posteroanterior chest radiograph demonstrating the fractured injection needle in the right ventricle.

of $8.4 \mathrm{Kpa}$ and $\mathrm{PCO}_{2}$ of $6.3 \mathrm{Kpa}$ on air. She was in respiratory distress but not feverish and haemodynamically stable. She had multiple scars at her wrist, antecubital fossa and both groins. A chest radiograph and computed tomography demonstrated a bilateral empyema, and a needle in the RV. Transthoracic and transoesophageal echocardiography showed the needle to be completely embedded in the wall of the RV and not suitable for transvenous retrieval. She refused surgical removal of the needle embolus. The bilateral empyema was treated by VATS drainage and decortication, and she was discharged six days after surgery.

\section{Discussion}

The retention of injection needles at the site of intravenous drug injection is an unusual occurrence not yet reported in injecting drug users. The repeated use of an injection needle and its manipulation to gain intravenous access at different sites can cause it to fracture. It is also possible that this may have been deliberately self inflicted, considering this patient's suicidal proclivity.

The development of DVT in the same leg can be explained by impaired venous return that can result from chronic inflammation and induration involving the femoral vein at the site of needle retention.

The embolisation of a fractured injection needle to the RV is a clinical rarity. Di Carlo et $a l,{ }^{3}$ and Kaushik and associates ${ }^{4}$ reported the embolisation of a fractured implantable venous catheter and a 0.38 calibre bullet to the right atrium, respectively. The entry of the fractured 
needle into the venous system through a large vein with less turbulent flow may have facilitated its embolisation to the RV. This probably prevented the needle from transgressing the vein en route to the RV.

Blood culture positive endocarditis in injecting drug users is usually due to staphylococcal aureus, and affects the right side of the heart mainly. ${ }^{12}$ The findings in this patient corroborate this. The needle embolus provided a focus for recurrent bacterial endocarditis.

Chronic local inflammatory response to, and the formation of vegetations on the needle, coupled with the contractile motion of the RV probably contributed to its incorporation into the RV wall. This clearly poses a management dilemma. Sometimes foreign bodies in the heart can be left alone, ${ }^{5}$ but as this case illustrates, it poses a high risk of recurrent bacterial endocarditis in people with increased susceptibility such as injecting drug users. There is also the potential risk of transmigration through the wall of the RV. Therefore, the removal of an RV needle embolus or any foreign body in the RV is desirable at a stage when transvenous retrieval can be achieved with low morbidity. Kaushik et $a l^{4}$ and

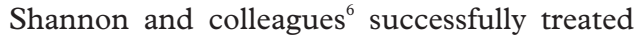
bullet emboli by early percutaneous removal.

An early retrieval of the needle embolus in this patient would have precluded the occurrence of other complications and eliminated the abiding potential risk of transmigration of the RV wall.

We wish to thank Mrs Chris Hillier, cardiothoracic secretary, Castle Hill Hospital, Cottingham, for her clerical assistance.

Funding: none.

Conflicts of interest: none.

1 DeWitt DE, Paauw DS. Endocarditis in injection drug users. Am Fam Physician 1996;53:2045-9.

2 Hecht SR, Berger M. Right-sided endocarditis in intravenous drug users. Prognostic features in 102 episodes. Ann Intern Med 1992;117:560-6.

3 Di Carlo I, Fisichella P, Russello D, et al. Catheter fracture and cardiac migration: a rare complication of totally implantable venous devices. F Surg Oncol 2000;73:172-3.

4 Kaushik VS, Mandal AK. Non-surgical retrieval of a bullet embolus from the right heart. Catheter Cardiovasc Interv 1999;47:55-7.

5 Kronson JW, Demetriades D. Retained cardiac missile: an unusual case report. $\mathcal{F}$ Trauma $2000 ; 48: 312-13$.

6 Shannon FL, McCroskey BL, Moore EE, et al. Venous bullet embolism: rationale for mandatory extraction. $\mathcal{F}$ Trauma 1987;27:1118-22. 